\title{
CIUDADANOS SOCIALMENTE COMPETENTES: Cómo trabajar la asertividad en educación infantil
}

\author{
Matilde PEINADO RODRÍGUEZ \\ Universidad de Jaén (España) \\ mpeinado@ujaen.es
}

SOCIALLY COMPETENT CITIZENS: How we work assertiveness in childhood education

Resumen: La formación en conductas asertivas ha sido, hasta fechas recientes, meramente testimonial en las aulas, cuando ésta presenta, a nuestro entender, enormes potencialidades en el proceso de socialización y en el ejercicio de la ciudadanía. En el presente trabajo trataremos de fundamentar, en primer lugar, que entendemos por asertividad, en segundo término cómo no sólo es posible, sino imprescindible, que se aborde desde la etapa infantil, pues su "naturalización" social y relacional puede mejorar la convivencia de las generaciones venideras; a continuación, trataremos de justificar su presencia en el currículum desde la competencia social y ciudadana y por último realizamos una propuesta de actividades para trabajar la asertividad con el alumnado de cinco años.

Abstract: Training in assertive behavior has been, until recently, merely testimonial in the classroom, where it has, to our knowledge, enormous potential in the process of socialization and exercise of citizenship. In this paper we try to justify, first, we understand as assertiveness, secondly how not only possible but essential, which is approached from the infant stage, as its social and relational "naturalization" can improve the coexistence of succeeding generations; then try to justify their presence in the curriculum from the social and civic competence and finally propose an assertive activities to work with students five years.

Palabras clave: Asertividad, competencia social y ciudadana, empatía, educación infantil. Assertiveness, social and civic competence, empathy, children education. 


\section{Introducción}

“...no es verdad que todo sucede después.

Es verdad exactamente lo contrario, es decir, todo sucede antes.

El largo periodo más importante de la vida, en el cual se asientan las bases sobre las que se construirán la personalidad, la cultura, las habilidades de la mujer y del hombre, es el de los primeros días, los primeros meses y los primeros años" Tonucci (2003: 57).

"La ciencia nos ha ayudado a tener el control increíble sobre nuestro mundo exterior, pero hemos avanzado poco en el control de nuestros mundos internos, emocionales" (Ramos, 2012). Con esta afirmación comienza una de las últimas investigaciones en inteligencia emocional, un nutrido elenco de publicaciones donde conviven estudios rigurosos con otros más divulgativos e incluso oportunistas, si bien todos ellos, comenzando por el ya clásico análisis de Goleman (1999) han reiterado una realidad que se nos muestra como obvia en la sociedad actual: las personas no hemos sido preparadas para entender y gestionar nuestras emociones, presentando problemas vitales y existenciales que padecemos y trasladamos a nuestro mundo relacional.

Este trabajo se inserta en la línea de investigación que entiende el proceso educativo como un desarrollo integral del individuo, y no sólo porque contempla las dimensiones intelectual y emocional de la persona, sino porque entiende que en un exitoso proceso de enseñanza-aprendizaje se fundamenta, en gran medida, en unas condiciones personales (familiares, afectivas, culturales, psicológicas) óptimas. La aparición de las competencias, con la llegada de la LOE, se inspira en este planteamiento, formar individuos competencialmente preparados para enfrentarse a los retos personales y sociales del mundo actual, incluso algunas comunidades autónomas, como Castilla la Mancha, han integrado la competencia emocional ${ }^{1}$ entre las básicas, pues sólo las personas competentes socialmente pueden construir y ejercer una ciudadanía activa y positiva.

El término positivo, en este contexto, tiene un sentido claramente ético y moral, asociado a lo que entendemos por bueno y al reconocimiento de otra praxis, la mala ciudadanía, "la ciudadanía enferma" que genera una convivencia perniciosa. Dicho argumento deriva de la propia reflexión de la autora en torno al mundo académico, que he tomado como pequeño laboratorio de análisis, de pulso social, la Universidad, cuya razón de ser, desde el Renacimiento, debiera ser "la transformación del hombre por obra de la ciencia y el saber" pero que, lejos de liderar un progreso humano basado en principios éticos, está entregada sin tapujos al vaivén competitivo, que no competencial, impuesto por los dictados economicistas, donde la cada vez más exigente formación intelectual no lleva aparejada una formación individual indispensable para una convivencia social y laboral sana.

En su seno, como un espejo de la realidad social, alberga profesionales con un alto grado de desadaptación, cuando el leiv motiv de una educación obligatoria, cada vez más dilatada en el tiempo es, en última instancia, garantizar la integración social del individuo, que debe asumir su responsabilidad en la consecución de un mundo mejor... ¿qué está pasando entonces?

1 Las competencias emocionales pueden definirse como un conjunto de conocimientos, capacidades, habilidades y actitudes necesarias para comprender, expresar y regular de forma apropiada los fenómenos emocionales (Bisquerra, 2003). 
Necesitamos, por tanto, una nueva visión del estudio de la inteligencia humana más allá de los aspectos cognitivos e intelectuales, que resalte la importancia del uso y la gestión del mundo emocional y social para comprender el curso de la vida de las personas, es decir, nos sitúan de nuevo ante la importancia de la formación en los tres principios básicos de la inteligencia emocional: a) Identificar mis estados anímicos (autoconomiento) y valorarme desde ellos (autoestima); b) Empatía: ser capaces de entender los sentimientos de los demás y c) Asertividad: expresar lo que pensamos y sentimos de forma saludable y no dañina para los demás.

Es evidente que los tres principios son insustituibles y están relacionados entre sí, pero consideramos que la asertividad se ha afrontado, hasta fechas recientes, en las aulas, de forma parcial y dependiente de la autoestima y la empatía, cuando ésta presenta, a nuestro entender, enormes potencialidades para la sociabilidad, por lo que el planteamiento, en las líneas que nos suceden, es tratar de fundamentar en primer lugar que entendemos por asertividad, en segundo término cómo no sólo es posible, sino imprescindible, que se aborde desde la etapa infantil, pues su "naturalización" social y relacional puede mejorar la convivencia de las generaciones venideras; a continuación, trataremos de justificar su presencia en el currículum desde la competencia social y ciudadana y por último realizamos una propuesta de actividades para trabajar la asertividad con el alumnado de cinco años.

\section{Hacia una conceptualización de la asertividad}

Lacalle Pereira (2009) define la asertividad como "la capacidad de hacer valer los propios derechos respetando también los de los demás, es decir, una habilidad que permite a las personas que la practican ser fieles a sí mismas en cualquier ocasión".

Por tanto, la asertividad no define a las personas sino a las conductas y actitudes, lo que encierra una disyuntiva: por una parte no podemos garantizar que las personas sean asertivas siempre, pero, desde el punto de vista educativo, en tanto que no se adscribe a al carácter, la personalidad o la herencia genética, sino a acciones, puede ser aprendida, como expone Gëll (2004) a partir de un entrenamiento.

Las respuestas asertivas son las más adecuadas para que los niños se desenvuelvan correctamente en sus relaciones sociales, pues es una habilidad que permite hacer multitud de cosas, desde formular una crítica, aceptar o recibir un halago, hasta reclamar algo o manifestar una queja, pero siempre desde la libertad y la independencia; la persona no se deja manipular ni busca manipular a otras personas.

Ahora bien, la clave de la asertividad como eje vertebrador del proceso de socialización es, sin duda, cómo prepara a las personas para expresar sus propios intereses, creencias, opiniones y deseos de un modo honrado, sin perjudicar ni agredir los deseos, intereses y sobre todo derechos de los demás, que podría concretarse en las siguientes pautas:

Decir " no" sin sentirse culpables.

-Saber expresar lo que sienten o piensan a los demás.

-Hablar diciendo la verdad sin necesidad de herir.

-Favorecer las relaciones con las personas.

-Aprender a hacer críticas positivas.

-Reconocer los derechos de uno mismo y los de los demás.

-Alcanzar los propios objetivos sin dañar a nadie.

Evidentemente, al movernos en el espacio de lo emocional, los límites entre autoestima, empatía y asertividad son difusos porque todos confluyen en la complejidad y riqueza del individuo, pero existen matizaciones importantes en la interpretación de los mismos, y, fundamentalmente, en la proyección social. Así, para saber expresar lo que sienten o piensan 
los demás, es necesario ponerse en el lugar del otro, empatizar, sentir como la otra persona, tratar de ver las cosas como el/ella las ve, pero ello no implica necesariamente utilizar dicho conocimiento en sentido positivo, mientras que la conducta asertiva lo canaliza hacia la apertura positiva, sin daño ni manipulación, por tanto, enriquece y perfecciona la empatía en pro del bien común.

Así, tener conciencia emocional, es decir, reconocer las emociones propias y las de los demás, nos permite disfrutar y fomentar las emociones agradables y ser capaces de regular y controlar aquellas que nos desagradan a nosotros y, consecuentemente a las demás, pues pueden convertirse en fuente de conflicto permanente, y que, latentes o soterrados en espacios y tiempos compartidos con otras personas, son el origen de la inmensa mayoría de los problemas que posteriormente surgen en el mundo laboral, familiar o social.

La asertividad, por tanto, mejora la convivencia, pues se define también como la capacidad para "ser fiel a uno mismo en cualquier ocasión", que, en el proceso de socialización, se interpreta como la capacidad para hacer valer los propios derechos respetando también los de los demás, un pilar fundamental para el ejercicio y práctica de la ciudadanía, pues los "derechos asertivos" están en la base y fundamento de los derechos humanos. Vamos a destacar aquellos que consideramos deberían impregnar el actual currículum educativo en Educación Infantil:

$1^{\circ}$ El derecho a ser tratados con respeto y respetar a los demás.

$2^{\circ}$ El derecho a decir “no” y no sentirnos culpables por ello.

$3^{\circ}$ El derecho a expresar nuestros propios sentimientos.

$4^{\circ}$ El derecho a tomarnos nuestro tiempo para parar, pensar, reflexionar y decidir.

$5^{\circ}$ El derecho a cambiar de opinión.

$6^{\circ} \mathrm{El}$ derecho a cometer errores.

\section{¿Por qué trabajar la asertividad desde la niñez?}

De los cuatro pilares de la educación (conocer, saber hacer, convivir y ser), señalados en el informe Delors (1996), como mínimo los dos últimos contribuyen a fundamentar la necesidad de la educación emocional en el marco de una educación integral desde la escuela.

Son muchos los autores que apuntan la necesidad de educar las habilidades sociales desde las primeras edades como un requisito necesario para una buena socialización posterior (Bisquerra, 2000; López ,2003; Monjas Casares, 2007; Gil, Mestre, González, y Foncubierta, 2011). Los niños nacen con predisposiciones emocionales específicas, pero, como expone Shapiro (2006: 25), su sistema de circuitos cerebrales retiene por lo menos cierto grado de plasticidad, por lo que pueden aprender nuevas capacidades emocionales y sociales que crearán nuevas vías nerviosas y pautas bioquímicas más adaptables

El desarrollo emocional y cognitivo de la persona progresan paralelamente; sin conocimiento no hay experiencia afectiva, y sin emociones no hay encuentro con la otredad, no se inicia el proceso de socialización, por lo que la alfabetización emocional es consustancial a la alfabetización intelectual; es, en definitiva, una educación para la vida.

Lantieri (2010: 35) va más allá al afirmar que, al menos en parte, el éxito académico depende de la capacidad del estudiante para mantener interacciones sociales positivas, y fundamenta dicha afirmación en el desarrollo de una serie de capacidades, donde la asertividad adquiere un papel relevante, de las que destacamos dos: a) toma de decisiones responsable considerando las consecuencias de las mismas para uno mismo y para los demás y b) rechazar presiones de los compañeros y mantener relaciones sanas y gratificantes con los individuos y con el grupo.

Ambas capacidades, que deberían erigirse en reglas de vida para la colectividad social, requieren una madurez intelectual y personal en la que seguimos evolucionando a lo largo 
de la vida, podríamos decir incluso que se trataría de un proyecto vital de carácter individual que nos capacita para el ejercicio de la sociabilidad y, como todo proyecto vital, debe empezar en la infancia. Dentro de la etapa de Educación Infantil, consideramos idóneo para trabajar la asertividad el tercer curso, teniendo en cuenta los siguientes aspectos:

- Argumentábamos con anterioridad cómo la asertividad enriquece y dirige positivamente la empatía y, por ello, debemos partir de las investigaciones llevadas a cabo sobre el desarrollo de la empatía, que sitúan en torno a los tres años la evolución desde la empatía egocéntrica, de la que continuarán presentando rasgos entre los tres y los seis años, hacia la empatía con los sentimientos de los demás, que comienza con el reconocimiento de que los otros pueden tener sentimientos diferentes a los propios.

- Es la etapa, siguiendo a Guil y otros (2011) en la que empiezan a copiar y asimilar los méritos de los demás, a identificarse con otras personas y, de esta forma, son capaces de ponerse en el lugar del otro y, al mismo tiempo, objetivizar cada vez mejor el mundo exterior.

- Díaz Navarro (2010: 5) afirma que es en torno a los cinco años cuando el alumnado, aun presentando las características narcisistas de la etapa, inicia sus tanteos socializadores, con su necesidad de juego y movimiento, con su autonomíacreciente, con suafianzamientodel lenguaje,pero,fundamentalmente, es cuando comienzan a elaborar, de una forma generalizada, algunos principios morales, a controlar sus impulsos y a integrar normas de comportamiento.

- A partir de esta edad, aunque puede iniciarse con anterioridad, comienzan a regular sus emociones, sin duda la clave para desarrollar conductas asertivas: 1)se abren a sentimientos tanto placenteros como desagradables, 2)comienzan a expresar sus emociones, por lo que es fundamental enseñarles desde el principio, y de una forma totalmente natural y normalizada cómo expresarlas sin dañar a los demás, 3) empiezan a entender las implicaciones de sus estados emocionales en su relación con los demás. Como afirma Lacalle Pedreira (2009: 10), es bueno que aprendan a expresar sus sentimientos, pero hay que hacerlo de la forma más adecuada, saludable para nosotros y no dañina para los demás.

\section{La asertividad en el currículum de Educación Infantil}

En nuestro país, como exponíamos al comienzo de este trabajo, se ha generado todo un movimiento educativo que aboga por introducir la educación de las emociones en el sistema, evidenciando la preocupación de los educadores por cambiar una escuela que se percibe como crisis e incapaz de afrontar los múltiples desafíos de nuestra sociedad (Fernández Berrocal, 2008: 427), pero el problema sigue siendo como reflejar dicha inquietud en el curriculum y, fundamentalmente, cómo llevarlo a la práctica.

En el trabajo coordinado por Ribes (2005) llevaron a cabo una propuesta pionera de currículum emocional en educación infantil (3-6 años) de la que recuperamos algunas principios metodológicos que hemos tenido en cuenta para elaborar una batería de actividades en torno a la asertividad en esta etapa: iniciación en el conocimiento y la conquista del mundo exterior y del otro y como adecuar mi actuación a la situación emocional de los demás; hacer especial uso de todas aquellas dinámicas que permitan la imitación de papeles para ser capaces de ponerse en el lugar de los otros; prestar atención al correcto desarrollo de las emociones de culpa y vergüenza o reconducir el uso de la agresividad en las interacciones.

Como exponen Feliu y Jiménez (2014: 382) aunque tradicionalmente se ha mantenido que los contenidos de Ciencias Sociales se encuentran vinculados al área de descubrimiento 
del entorno de educación infantil, el actual curriculum apuesta por aprendizajes globalizados, estableciendo relaciones entre los contenidos de las diferentes áreas, a fin de acercarles a la interpretación del mundo, dándole significado y facilitándoles la participación activa. La formación en conductas asertivas, en tanto que clave para la consecución de la competencia social y ciudadana es transversal, está presente tanto en los objetivos generales, especialmente en el objetivo siete (identificar los propios sentimientos, emociones y necesidades y comunicarlos a los demás así como identificar y respetar las de los demás) como en los contenidos del bloque primero del Área de Conocimiento del Entorno, "El cuerpo y la propia imagen", donde se refleja como contenido conceptual el reconocimiento de los sentimientos y emociones propios y de los demás y saber manifestar y regular progresivamente sentimientos, emociones, preferencias, vivencias e intereses.

Volvemos sobre la investigación de Ribes (2005) para completar el currículum desde la educación emocional y su implicación en el desarrollo de la moralidad: las emociones morales. Define la educación emocional (Ibídem, 2005: 5) como un proceso educativo, continuo y permanente, que pretende potenciar el desarrollo de las competencias emocionales como elemento esencial del desarrollo integral de la persona, con objeto de capacitarle para la vida y cuya finalidad esencial es aumentar el bienestar personal y social, minimizando la vulnerabilidad de la persona ante determinadas disfunciones (estrés, depresión, impulsividad, agresividad, etc). Las emociones morales afloran y se educan a lo largo de toda la infancia y adolescencia. En esta etapa vamos a centrarnos, siguiendo a Vallés Arándiga y Vallés Tortosa (2000: 67) en dos:

-Adoptar y comprender lo que distingue una conducta "buena" de una "mala" y desarrollar hábitos de conducta compatibles con lo que perciben como "bueno".

-Desarrollar interés y consideración por el bienestar de los demás.

La finalidad última de dicho proceso formativo es, como no podía ser de otra forma, la capacitación social del alumnado, es decir su formación en habilidades sociales para establecer relaciones sociales satisfactorias, como potencial individual, que contribuirá a su éxito y satisfacción en la vida, y como agente social.

En efecto, desde el ámbito personal, para afrontar con garantías el proceso de socialización, toda persona debe aprender a reconocer, interpretar y responder en forma apropiada a las situaciones sociales, conciliando sus necesidades y expectativas con las de los demás.

En tanto que miembro de una comunidad, la gestión de las emociones está directamente

relacionada con el grado de socialización del niño/a y un inadecuado control afecta tanto a la adaptación escolar como a la social. Como exponen Morales, Trianes e Infante (2013: 56) la huella de una ciudadanía responsable en las actuaciones socioprofesionales no se logra de forma natural sino que requiere de aprendizajes y espacios que favorezcan la construcción ética de la persona: a partir de una ciudadanía responsable es posible evolucionar hacia mayores cotas de cohesión social.

\section{La asertividad en la construcción y ejercicio de la ciudadanía}

Entre la población adulta que nos rodea, empezando quizá por nosotros/as mismos, existen carencias significativas en la construcción de la personalidad, incluso se acuña el término de "analfabetismo emocional" para referirse a la carencia de herramientas para relacionarnos emocionalmente con los demás, para expresar verbal o corporalmente los sentimientos y afectos, para entender al otro, para relacionarnos en el tejido asociativo o laboral, una situación que se agrava, teniendo en cuenta el sesgo de género que aún caracteriza la cotidianidad de las aulas y las familias, en el colectivo masculino. 
Este "analfabetismo", con independencia de los evidentes problemas que provoca a nivel personal, se convierte en materia de análisis y revisión cuando genera, como venimos argumentando, problemas serios en el proceso de socialización: en las relaciones personales, en las relaciones de pareja, en el mundo laboral o en cualquier manifestación del tejido asociativo de una comunidad; se trata, por tanto, de herramientas indispensables en la construcción de la ciudadanía, siendo la asertividad la que presenta una dimensión social más acentuada. La esencia de la conducta asertiva puede ser reducida a cuatro patrones específicos: la capacidad de decir que no, la capacidad de pedir favores y hacer requerimientos; la capacidad de expresar sentimientos positivos y negativos, de forma directa, honesta y apropiada y la capacidad de iniciar, continuar y terminar conversaciones.

En el ejercicio de la ciudadanía responsable podemos distinguir dos facetas: la primera que parte del respeto a la integridad de las personas y del reconocimiento, en tanto que ciudadanos, de unos derechos inalienables a todos los individuos, en base a su condición de persona; dicha integridad, desde la óptica asertiva, se extiende más allá de este reconocimiento, al entender a las personas como sujetos con sentimientos que merecen no sólo ser destinatarios de un mensaje, sino la transmisión adecuada del mismo, tanto en las formas verbales como no verbales (mirada, gestos, tonos de voz, actitudes...), qué se dice y cómo se dice, una habilidad social que facilita y mejora sensiblemente la comunicación en foros formales y no formales.

La segunda faceta es la que se centra en la responsabilidad de las propias acciones, y derivado de ello, las consecuencias positivas y negativas de sus actos para otras personas. Como defiende Gell (2004: 15) suele decirse que con la asertividad cada uno puede conseguir lo que considere mejor para él y, al mismo tiempo, lo que considere más justo, ya que siempre tiene en cuenta los derechos de los demás.

La formación en derechos asertivos debería extenderse de manera sistemática y progresiva a lo largo de toda la educación obligatoria. Teniendo en cuenta la madurez intelectual y afectiva que, siempre en términos generales, presenta el alumnado de cinco años, vamos a trabajar, siguiendo la clasificación planteada por Monjas Casares (2007) los siguientes:

$1^{\circ}$ Puedes cambiar de opinión si te sientes incómodo.

$2^{\circ}$ Cuando comentes un error puedes admitirlo sin avergonzarte.

$3^{\circ}$ No tienes que gustarte lo que todo el mundo hace.

$4^{\circ}$ No es necesario que seas perfecto/a y no tienes por qué sentirte mal cuando eres simplemente tú.

Los maestros y maestras de Infantil son los encargados de acompañar a los niños y niñas en la construcción de su primera identidad y en el crecimiento social a partir del conocimiento de uno mismo y de los demás, pero como han analizado Torruella y Jiménez (2014: 380) el grado de Educación Infantil no contempla ninguna enseñanza vinculada al desarrollo de la inteligencia personal e interpersonal.

Los maestros y maestras, tanto en formación como en activo, necesitan formación continua para incorporar recursos que ayuden a desarrollar las competencias emocionales del alumnado, como, volviendo sobre el tema que nos ocupa, el desarrollo de conductas asertivas. Por ello desarrollamos a continuación un cuadrante de actividades fundamentadas tanto en recursos tradicionales como en otros presentes en la red ya que nuestra propuesta no parte de una presentación original e inédita de actividades, sino de dotar a las mismas de unos objetivos y contenidos orientados al desarrollo de conductas asertivas de una manera naturalizada, de forma que al tiempo que adquieren competencias que los capaciten para la socialización, las relaciones que establezcan con los demás estén fundadas desde su origen en la asertividad. 


\begin{tabular}{|c|c|c|c|c|c|c|c|c|c|}
\hline & & 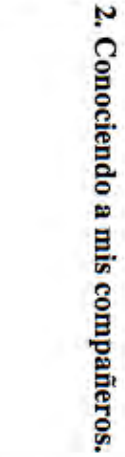 & & & & 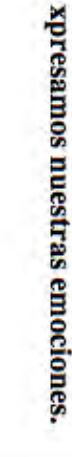 & 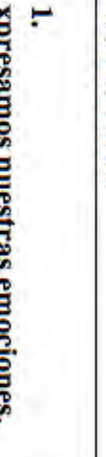 & 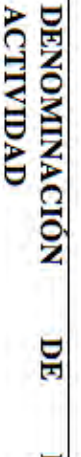 & 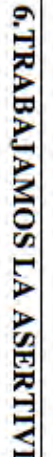 \\
\hline & & 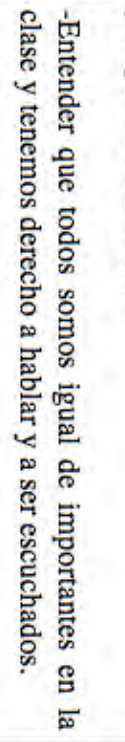 & 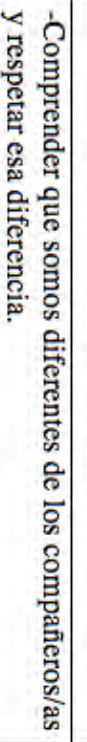 & & 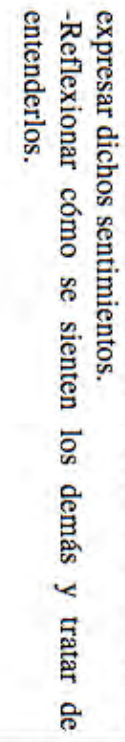 & 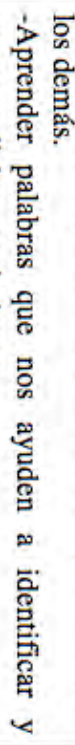 & 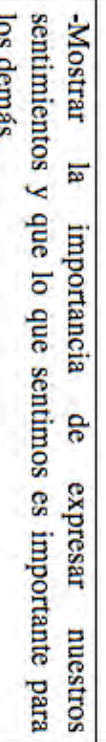 & 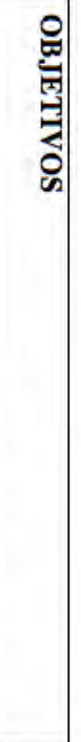 & \\
\hline 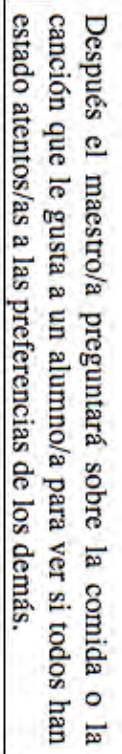 & 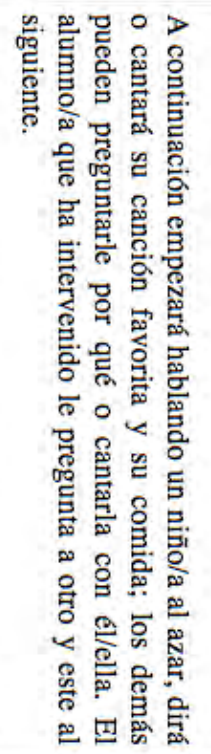 & 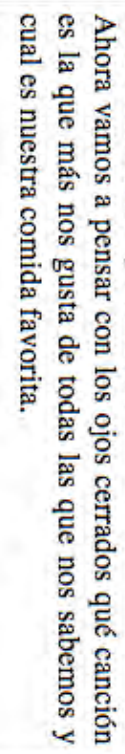 & 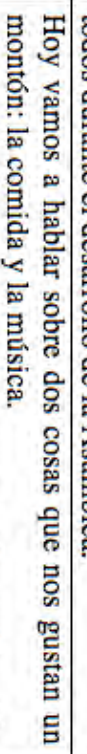 & 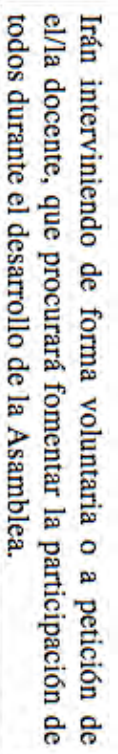 & 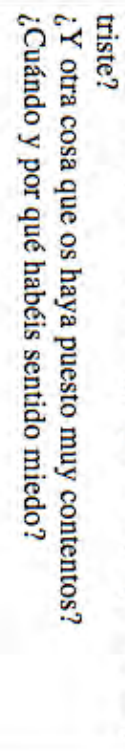 & 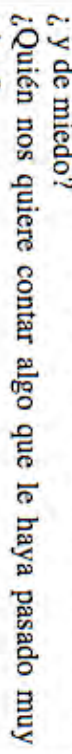 & 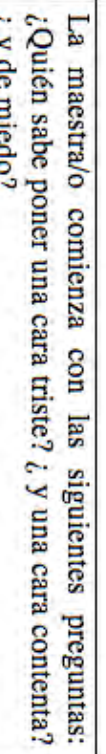 & 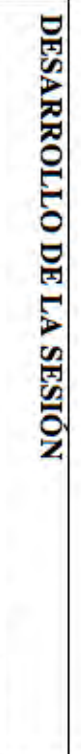 & \\
\hline
\end{tabular}




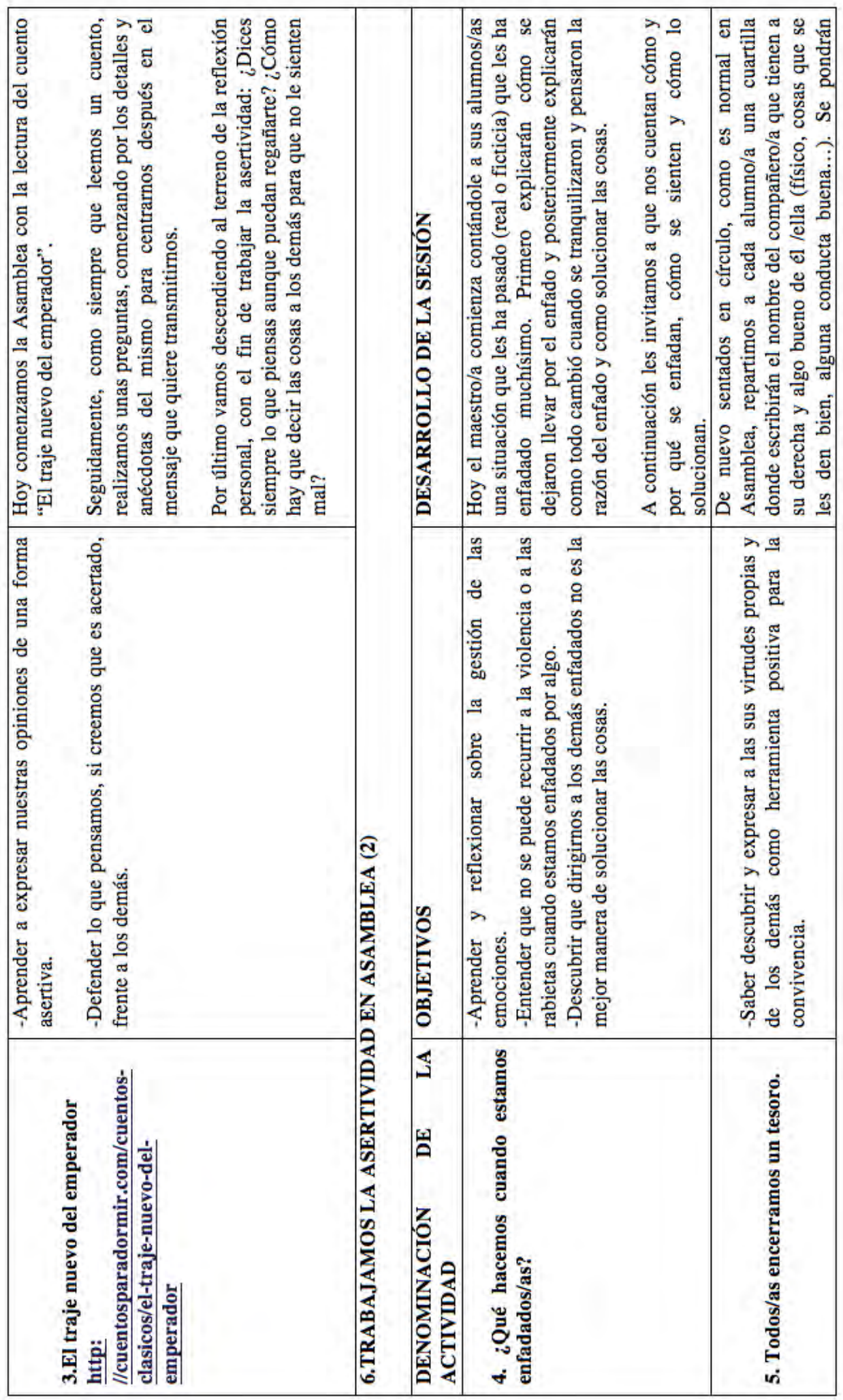




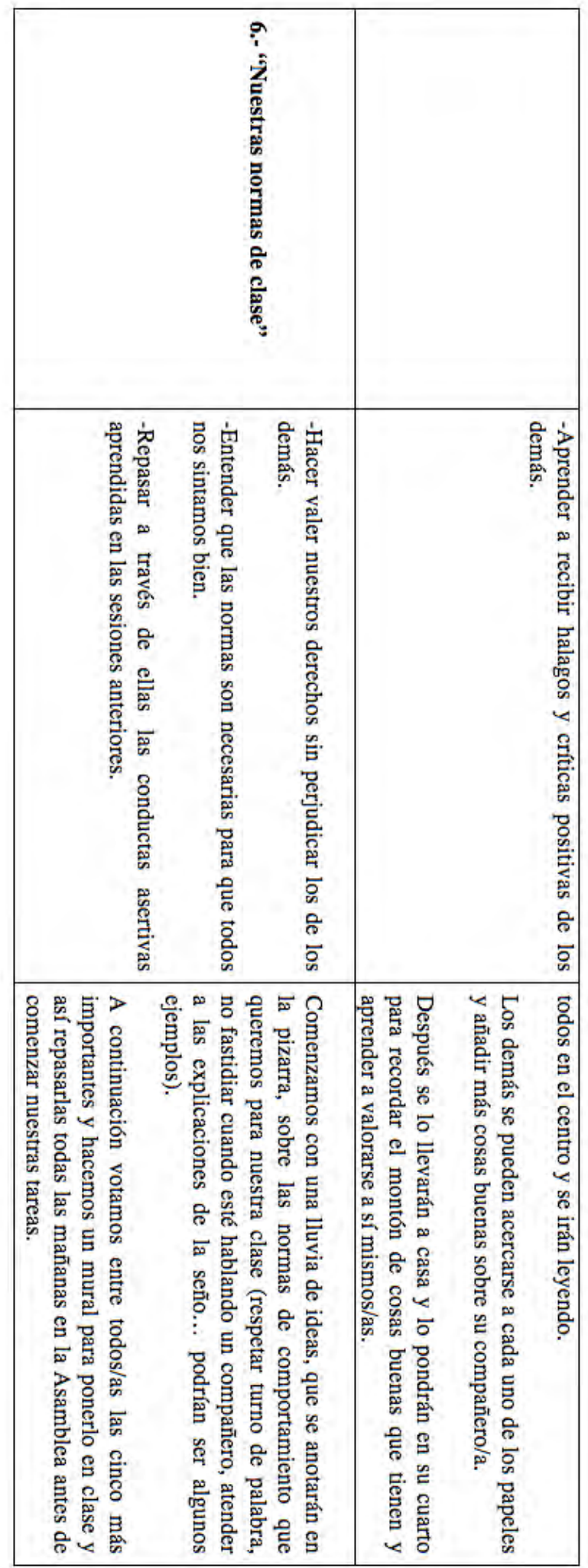




\section{Concluyendo}

En este trabajo hemos tratado de demostrar cómo la etapa de infantil es el momento idóneo para iniciar al alumnado, de una forma naturalizada y cuasi intuitiva, en la asertividad. Desde el ámbito individual, les permite conocer, disfrutar y gestionar sus emociones en aras de lograr mayores cotas de bienestar personal. Atendiendo al ámbito relacional, las conductas asertivas se erigen en una herramienta clave en la formación ética de la persona y su contribución a una convivencia saludable (familiar, laboral, vecinal, etc). En cuanto al proceso formativo, un exitoso proceso de enseñanza-aprendizaje se fundamenta, en gran medida, en una formación integral, tanto intelectual como emocional, óptima. Por último, atendiendo al fin último del sistema educativo, a la esencia de la escuela, esto es, la formación de ciudadanos corresponsables en la construcción de un mundo mejor, entender las relaciones con el otro/a desde el respeto pudiera ser la clave hacia la que proyectar, desde la infancia, una sociedad futura más libre, justa y democrática.

\section{Bibliografía}

AA. VV. (2013): Guía para mejorar la inteligencia emocional en los adolescentes. Madrid: Pirámide.

Feliu Torruella, M y Jiménez Torregosa, L. (2014): ¿Cómo somos? El descubrimiento de uno mismo y la construcción de la identidad en la formación del profesorado en Educación Infantil en $\mathrm{Vv}$. Aa. Una mirada al pasado y un proyecto de futuro: investigación e innovación en didáctica de las ciencias sociales. Barcelona: Universidad Autóma.

Fernández-Abascal, E (2009): Emociones positivas. Madrid: Pirámide.

Fernández Berrocal, P. (2005): Corazones inteligentes. Barcelona: Kairós.

Fernández Berrocal, P y Ruiz Aranda, D. (2008): La inteligencia emocional en la Educación. Revista Electrónica de Investigación Psicoeducativa, 15, 421-436.

Galindo, A. (2004): Inteligencia emocional para jóvenes. Madrid: Pearson.

Gëll, M. (2004): ¿Por qué he dicho blanco si quería decir negro? Técnicas asertivas para el profesorado y los formadores. Barcelona: Graó.

Gil, R; Mestre, J.M; González, G y Foncubierta, S (2011). Integración del desarrollo de competencias emocionales en el currículum de Educación Infantil. Reifop, 14 (3).

Goleman, D. (1999): La inteligencia emocional. Barcelona: Kairós.

- (2012): El cerebro y la inteligencia emocional. Barcelona: Ediciones B.

Ibarrola, B. (2003): Cuentos para sentir. Educar las emociones. Madrid: S.M.

-(2004): Educar los sentimientos. Madrid: S.M.

-(2005): Sentir y pensar. Programa de Inteligencia Emocional para niños y niñas de 3 y 5 años. Madrid: S.M.

Lacalle Pedreira, V. (2009): Inteligencia emocional, niños y adolescentes. Madrid: Gesfomedia.

Lantieri, L. (2010): Inteligencia emocional infantil y juvenil: ejercicios para cultivar la fortaleza interior en niños y jóvenes. Madrid: Aguilar.

Monjas Casares, M.I. (2007): Cómo promover la convivencia: programa de asertividad y habilidades sociales. Madrid: Cepe.

Ramos, N; Enriquez. H y Recondo, O. (2012): Inteligencia emocional plena. Madrid: Kairós.

Ribes, R; Buisquerra, M, Agulló, M.J, Filella, G y Soldevilla, A. (2005): Una propuesta de curriculum emocional en Educación Infantil (3-6 años). Cultura y Educación , 17 (1), 5-17.

Shapiro, L. (2006): La inteligencia emocional de los niños. Una guía para padres y maestro. Barcelona: Byblos.

Tonucci, F. (2003): Cuando los niños dicen ¡Basta!, Madrid: Fundación Germán Sánchez Ruipérez.

Vallés Arándiga, A. y Vallés Tortosa, C. (2000): Inteligencia emocional. Aplicaciones educativas. Madrid: Eos. 